05

\title{
Влияние токового отжига на температурные зависимости магнитоимпеданса в аморфных микропроводах
}

\author{
() А. Джумьазода, ${ }^{1}$ Л.В. Панина, ${ }^{1,2}$ М.Г. Неьматов, ${ }^{1}$ Н.А. Юданов, ${ }^{1}$ Ф.С. Табаров, ${ }^{1}$ А.Т. Морченко, ${ }^{1}$ \\ A.A. Ухасов $^{1}$ \\ ${ }^{1}$ Национальный исследовательский технологический университет „МИСиС“, \\ 119049 Москва, Россия \\ ${ }^{2}$ Институт проблем проектирования в микроэлектронике РАН, \\ 124681 Москва, Россия \\ e-mail: abdukarim_jumaev@mail.ru
}

Поступило в Редакцию 29 ноября 2018 г.

В окончательной редакции 29 ноября 2018 г.

Принято к публикации 29 января 2019 г.

\begin{abstract}
Миниатюризация устройств магнитной электроники и микросистемной техники во многом зависит от оптимального выбора функциональных материалов (сред), используемых в качестве рабочего тела, в частности, чувствительных элементов различных сенсорных систем (например, датчиков локальных магнитных полей, механических напряжений и/или деформаций, температуры и т.п.). Одним из перспективных материалов являются ферромагнитные микропровода, состоящие из жилы аморфных сплавов в стеклянной оболочке, проявляющие высокую чувствительность магнитоимпеданса (МИ) к изменению указанных внешних факторов - эффект гигантского МИ (ГМИ). При этом для множества приложений важным является температурная стабильность рабочей характеристики таких устройств.
\end{abstract}

DOI: 10.21883/JTF.2019.07.47798.411-18

В настоящей работе проведено всестороннее исследование влияния температуры на поведение магнитоимпеданса в аморфных ферромагнитных микропроводах $\mathrm{Co}_{60.51} \mathrm{Fe}_{3.99} \mathrm{Cr}_{12.13} \mathrm{~B}_{13.53} \mathrm{Si}_{9.84}$. Сплавы подобного состава имеют относительно высокую точку Кюри $\left(T_{C}>300^{\circ} \mathrm{C}\right)$, однако изготовленные на их основе микропровода зачастую проявляют значительную зависимость магнитных свойств даже вблизи комнатной температуры (ниже $100^{\circ} \mathrm{C}$ ). Для обеспечения термостабильности процессов намагничивания и магнитоимпеданса в широком диапазоне температур проводилась термообработка микропроводов за счет теплового действия постоянного тока.

\section{Введение}

Точка Кюри - важнейшая характеристика магнитных материалов, применяемых в устройствах современной магнитоэлектроники и микросистемной техники в качестве рабочей среды/тела. В частности, она во многом определяет температурное поведение большинства магнитных свойств, и, как следствие, диапазон рабочих температур и термостабильность функционирования устройств, построенных на основе этих материалов. Ферромагнитные сплавы $3 d$ металлов с добавлением других элементов (разбавителей и/или аморфизаторов), получаемые в виде изделий пониженной размерности: тонких пленок, проволок, микро- и нанопроводов, являются объектом особо пристального изучения многочисленных научных коллективов.

С практической точки зрения наиболее подходящими элементами для использования в сенсорных системах в настоящее время представляются аморфные ферромагнитные микропровода (АФМП) благодаря их специфическим анизотропным характеристикам $[1,2]$. При наличии механических напряжений за счет различия коэффициентов термического расширения материалов жилы и оболочки в проводах с близкой к нулю положительной или отрицательной константой магнитострикции формируется наведенная механическими напряжениями магнитная анизотропия: продольная - c направлением легкого намагничивания вдоль оси провода или специфического вида (циркулярная или геликоидальная) [3]. Вид реализующейся магнитной структуры проводов определяет характер и динамику процессов намагничивания, форму петли гистерезиса. Более того, многие свойства таких проводов зависят от внешних факторов (например, механического напряжения, деформации, температуры) [4-6]. Это позволяет разрабатывать на основе таких проводов различные датчики, в которых они исполняют роль чувствительного элемента $[7,8]$.

Свойствами материала можно управлять также в зависимости от способов модифицирования, используемых после изготовления микропроводов. Так, в ряде работ, посвященных АФМП, подвергнутым последующей термообработке в различных условиях, отмечалось сильное влияние режима отжига на механические параметры, намагниченность, магнитную анизотропию, магнитострикцию, коэрцитивную силу, точку Кюри, электросопротивление и др. характеристики микропроводов [9-16].

Термический отжиг в присутствии магнитного поля, который можно легко применить к АФМП или всему МИ сенсорному элементу, представляет большой тех- 
нологический интерес для повышения чувствительности датчика без изменения его конструкции. В этом контексте следует отметить, что управление свойствами микропровода за счет уменьшения магнитоупругой связи путем тщательного выбора состава сплава и последующего токового отжига позволяет достичь максимального значения МИ (до $600 \%$ на частоте $15 \mathrm{MHz}$ ) [17].

Изучение воздействия отжига на петлю гистерезиca, коэффициент магнитострикции и эффекта ГМИ в АФМП существенно важно для понимания процессов, влияющих на магнитную мягкость и, как следствие, для улучшения магнитных характеристик, в том числе усиления эффекта ГМИ [18]. С другой стороны, основой устойчивого и надежного функционирования служит температурная стабильность магнитных свойств материала сенсорного элемента. В связи с этим в настоящей работе проводились исследования влияния условий отжига на температурную зависимость магнитных свойств микропроводов номинального состава $\mathrm{Co}_{60.51} \mathrm{Fe}_{3.99} \mathrm{Cr}_{12.13} \mathrm{~B}_{13.53} \mathrm{Si}_{9.84}$, которые обладают чрезвычайно малой магнитострикцией в исходном состоянии и обычно считаются наиболее перспективными для использования в приложениях, основанных на эффекте ГМИ.

Зависимость магнитных свойств от температуры объясняется тем, что во время нагрева происходит релаксация внутренних механических напряжений, наведенных в процессе производства микропровода, присутствие которых нежелательно в сенсорных системах с применением МИ. С другой стороны, в аморфных проводах с относительно большой магнитострикцией и низкой точкой Кюри $(T)[19]$ температурную зависимость МИ можно контролировать и использовать ее для локализованных измерений температуры окружающей среды. Таким образом, на основе сплавов почти одинакового химического состава можно осуществить получение широкой гаммы микропроводов, чувствительных к изменению температуры, за счет управления значением $T_{\mathrm{C}}$ в диапазоне от комнатной температуры до $\sim 300^{\circ} \mathrm{C}$.

Как сообщалось ранее [20], во время токового отжига микропровод подвергается как нагреву, так и воздействию кругового магнитного поля тока, совместное действие которых наводит циркулярную магнитную анизотропию, наиболее заметную в периферийной части провода, что обеспечивает усиление эффекта ГМИ.

Недавно было изучено влияние таких параметров, как частота, амплитуда и направление магнитного поля во время токового отжига, на ГМИ и магнитные свойства проводов на основе кобальта $[21,22]$. Было обнаружено, что в результате отжига в микропроводах появляется сложная доменная структура. В связи с этим в наших экспериментах процесс термообработки проводился таким образом, чтобы параметры отжига и температура окружающей среды способствовали достижению компромисса между чувствительностью МИ-эффекта в проводе и термостабильностью магнитных характеристик провода. В настоящей работе мы исследовали температурную зависимость ГМИ в микропроводах, взятых в исходном состоянии и подвергнутых отжигу путем пропускания постоянного электрического тока $25 \mathrm{~mA}$ в течение $60 \mathrm{~min}$ на сравнительно низки и высоких частотах (40 и $300 \mathrm{MHz}$ ).

\section{1. Детали экспериментального исследования}

Было изучено влияние температуры на эффект ГМИ в аморфных микропроводах номинального состава $\mathrm{Co}_{60.51} \mathrm{Fe}_{3.99} \mathrm{Cr}_{12.13} \mathrm{~B}_{13.53} \mathrm{Si}_{9.84}$ со стеклянной оболочкой, изготовленных методом Тейлора-Улитовского [23] и имеющих общий диаметр $24.7 \mu \mathrm{m}$. Температура Кюри проводов составляла выше $300^{\circ} \mathrm{C}$.

Обработка проводов осуществлялась электрическим током величиной $25 \mathrm{~mA}$, подаваемым от стабилизированного источника постоянного напряжения. Электрический ток воздействовал на микропровод длиной $15 \mathrm{~cm} \mathrm{в} \mathrm{течение} 60 \mathrm{~min}$. Для фиксации проводника в прямолинейном положении во время прохождения тока использовался специальный держатель. Концы провода крепились к держателю с оловянным припоем.

Магнитоимпеданс проводов определялся на основе измерения параметра S12 с помощью векторного анализатора цепей НР $8753 \mathrm{E}$ в широком диапазоне частот (1-300 MHz). Провод помещался в специально разработанную микрополосковую ячейку (держатель образца). Для создания магнитного поля напряженностью \pm 20 Ое использовались катушки Гельмгольца. Измерительный блок объединял в единой конструкции намагничивающие катушки с контейнером для фиксации микрополосковых ячеек и термоизолированной камеры. Ячейка подключалась к портам передатчика/приемника установки. Образец провода длиной $1 \mathrm{~cm}$, припаянный к контактным площадкам измерительной ячейки, возбуждался сигналом источника синусоидального напряжения. Амплитуда переменного тока возбуждения в проводе составляла 2-3 mA. Температура в камере варьировала от комнатной до $90^{\circ} \mathrm{C}$ и измерялась стандартной термопарой.

После обработки экспериментальных данных было проведено сравнение результатов измерения импеданса проводов до и после токовой обработки.

\section{2. Результаты (анализ) и обсуждение}

\section{1. МИ-свойства исходных проводов}

На рис. 1, $a$ показаны графики зависимости МИ исходного микропровода от магнитного поля $Z(H)$ на частоте $40 \mathrm{MHz}$ для различных температур (от комнатной температуры до $\left.90^{\circ} \mathrm{C}\right)$. Кривые МИ-проводов до и после обработки в диапазоне температур до $70^{\circ} \mathrm{C}$ имеют форму двойного пика с минимумом, соответствующим отсутствию магнитного поля. С повышением 

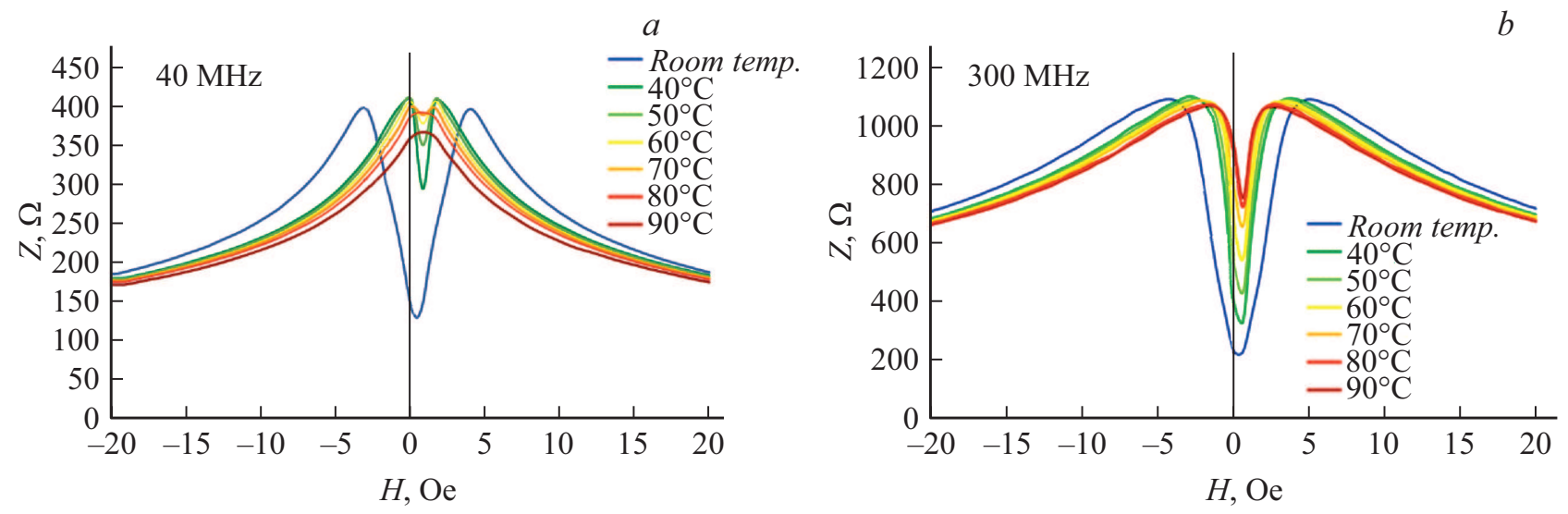

Рис. 1. МИ в исходном микропроводе $\mathrm{Co}_{60.51} \mathrm{Fe}_{3.99} \mathrm{Cr}_{12.13} \mathrm{~B}_{13.53} \mathrm{Si}_{9.84}$ при различной температуре: $a-$ на частоте $40 \mathrm{MHz} ; b-\mathrm{Ha}$ частоте $300 \mathrm{MHz}$.

температуры чувствительность импеданса к магнитному полю в области низких полей значительно уменьшается. Увеличение температуры выше $70^{\circ} \mathrm{C}$ приводит к кардинальной перестройке графиков МИ с появлением одного центрального пика вблизи нулевого значения магнитного поля (колоколообразная форма зависимости наблюдается, например, при температурах 80 и $90^{\circ} \mathrm{C}$ ). Изменение поведения МИ связано с преобразованием характера магнитной анизотропии от циркулярной к осевой из-за релаксации внутренних напряжений. Уменьшение МИ-эффекта с ростом температуры при $H=0$ огромно и составляет более $200 \%$ при $80^{\circ} \mathrm{C}$, что является неприемлемым при использовании таких проводов в качестве магниточувствительных элементов в датчиках магнитных полей.

На рис. 1, $b$ показано влияние температуры на МИ при более высоких частотах (до $300 \mathrm{MHz}$ ). В этом случае наблюдаются весьма высокая чувствительность ГМИ, велико также и изменение импеданса с температурой (до $250 \%$ при $90^{\circ} \mathrm{C}$ ), однако однопиковый импеданс при нагреве не возникает. Поскольку на более высоких частотах вклад в ГМИ дает приповерхностный слой металлической жилы АФМП, то можно предположить, что направление легкого намагничивания здесь сохраняет спиральную ориентацию и при более высоких температурах, хотя ее значение константы циркулярной/геликоидальной анизотропии непрерывно уменьшается с температурой. Таким образом, можно с уверенностью постулировать пригодность проводов исследуемого состава для построения высокочастотных сенсорных устройств, но при этом требуется обеспечить температурную стабильность их характеристик.

\section{2. МИ-свойства отожженных проводов}

Поведение ГМИ-проводов, отожженных действием постоянного тока, нами исследовалось на разных частотах в связи с возможностью индуцирования в них циркулярной анизотропии в процессе обработки и дости- жения повышенной чувствительности МИ. Что касается эффектов токового отжига, важно заметить, что магнитная структура АФМП в стеклянной оболочке такова, что центральная часть жилы, имеющая осевое направление вектора намагниченности, окружена приповерхностной областью с циркулярным или геликоидальным распределением намагниченности. Как правило, в литых проводах внутренняя область составляет основную часть объема провода. Поверхностная область с циркулярной анизотропией может быть расширена в процессе токового отжига путем индуцирования дополнительной анизотропии в присутствии кругового магнитного поля тока за счет образования и упорядочения магнитоактивных пар атомов.

Из зависимости импеданса от магнитного поля при различных температурах, приведенных на рис. 2, можно сделать вывод, что отжиг АФМП постоянным током приводит к значительному повышению температурной стабильности импеданса в диапазоне частот $1-300 \mathrm{MHz}$. Например, в отсутствие магнитного поля АФМП не проявляют какой-либо температурной зависимости импеданса в диапазоне от комнатной температуры до $90^{\circ}$. Эта устойчивость к повышению температуры может быть объяснена тем, что максимально возможная релаксация механических напряжений, наведенных в процессе изготовления АФМП, уже была достигнута в процессе токового нагрева, а круговое магнитное поле, создаваемое током, усилило циркулярную анизотропию. Так, в результате отжига положение пиков МИ сдвинулось с $5 \mathrm{Oe}$, наблюдаемых перед обработкой, до 8 Ое после нее; соответственно расширился и диапазон максимальной чувствительности МИ к изменению магнитного поля. Тем не менее при этом незначительно снижается чувствительность импеданса примерно с 55 до 40\% Ое в области низких полей (рис. 2, а для $40 \mathrm{MHz}$ ). Это связано с тем, что максимальное значение импеданса зависит от распределения легких направлений магнитной анизотропии в АФМП, для которого характерно уменьшение после токового отжига объема его центральной 

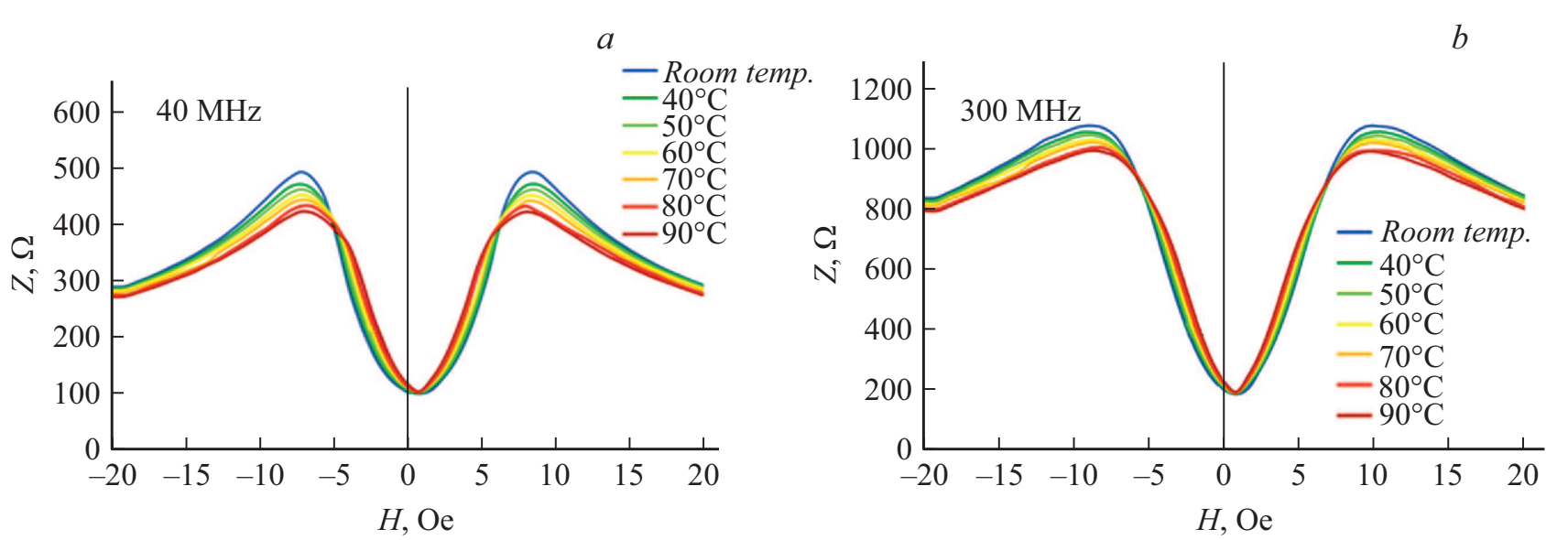

Рис. 2. Зависимость МИ от величины магнитного поля для отожженных микропроводов с номинальным составом $\mathrm{Co}_{60.51} \mathrm{Fe}_{3.99} \mathrm{Cr}_{12.13} \mathrm{~B}_{13.53} \mathrm{Si}_{9.84}$ для разных температур и частот: $a-40 ; b-300 \mathrm{MHz}$.

части с преимущественно аксиальным направлением намагниченности.

Аналогичный вывод можно сделать относительно поведения МИ-отожженных проводов на более высоких частотах, что видно из рис. 2, b. Важно отметить значительное увеличение чувствительности с увеличением частоты (правда, несколько более низкое, чем для неотожженных проводов) наряду с высокой температурной стабильностью. Таким образом, незначительное влияние температуры на ГМИ не приведет к снижению возможностей применения микропроводов в промышленных устройствах, принцип действия которых основан на использовании МИ-эффекта. После токового отжига чувствительность ГМИ на относительно высоких частотах может быть весьма большой, при этом она остается термостабильной в области всех исследованных частот для обычных режимов эксплуатации, и как ожидается, она не должна сильно зависеть от условий окружающей среды при повышенных или пониженных температурах.

\section{Выводы}

Исследована температурная зависимость магнитоимпеданса аморфных микропроводов состава $\mathrm{Co}_{60.51} \mathrm{Fe}_{3.99} \mathrm{Cr}_{12.13} \mathrm{~B}_{13.53} \mathrm{Si}_{9.84}$ в стеклянной оболочке до и после отжига в постоянном токе с целью достижения подходящего баланса между чувствительностью и температурной стабильностью ГМИ-эффекта. Сигнал ГМИ на низких и высоких частотах в диапазоне $1-300 \mathrm{MHz}$ продемонстрировал увеличение чувствительности с ростом частоты, однако наиболее значительное влияние нагрева наблюдалось на высоких частотах. Токовый отжиг приводит к возникновению четко выраженной круговой магнитной анизотропии; причем не было отмечено значительного влияния на нее температуры вплоть до $90^{\circ} \mathrm{C}$. Такие провода будут полезны для создания промышленных датчиков магнитного поля.
Показано, что из-за внутренней релаксации напряжений микропровода демонстрируют заметное изменение магнитной анизотропии от циркулярного типа к аксиальному. Это сопровождается огромным изменением магнитоимпедансного поведения - вид кривых МИ трансформируется от двух симметричных пиков до одного центрального пика.

\section{Благодарности}

Авторы благодарны В. Ларину, MFTI Ltd (http://www.microwires.com) за предоставление образцов микропроводов.

\section{Финансирование работы}

Работа выполнена при финансовой поддержке Министерства образования и науки Российской Федерации в рамках программы повышения конкурентоспособности НИТУ „МИСиС“, осуществляемой постановлением правительства от 16 марта 2013 г. № 211.

\section{Список литературы}

[1] Vazquez M., Chiriac H., Zhukov A., Panina L., Uchiyama T. // Phys. Stat. Sol. A. 2011. Vol. 208. P. 493.

[2] Qin F., Peng H.-X. // Prog. Mater. Sci. 2013. Vol. 58. P. 183.

[3] Panina L., Mohri K. // Appl. Phys. Lett. 1994. Vol. 65. P. 1189.

[4] Mandal K., Puerta S., Vazquez M., Hernando A. // IEEE Trans. Magn. 2002. Vol. 36. P. 3257.

[5] Popov V.V., Berzhansky V.N., Gomonay H.V., Qin F.X. // J. Appl. Phys. 2013. Vol. 113. P. 326.

[6] Makhnovskiy D., Zamorovski V. // J. Summerscales. Composites. A. 2014. Vol. 61. P. 216.

[7] Ripka P., Tipek A. Modern Sensors Handbook, Wiley-ISTE, 2007.

[8] Grosz A., Haji-Sheikh M.J., Mukhopadhyay S.C. High Sensitivity Magnetometers, Smart Sensors. Vol. 19: Measurement and Instrumentation, Springer International Publishing, Cham, Switzerland. 2017. 
[9] Kraus L., Knobel M., Kane S.N., Chiriac H. // J. Appl. Phys. 1999. Vol. 85. P. 5435.

[10] Liu J., Qin F., Chen D., Shen H., Wang H., Xing D., Phan M.H., Sun J. // J. Appl. Phys. 2014. Vol. 115. P. 326.

[11] Zhukova V., Talaat A., Ipatov M., Zhukov A. // IEEE Trans. Magn. 2014. Vol. 50. P. 1.

[12] Morchenko A.T., Panina L.V., Larin V.S., Churyukanova M.N., Salem M.M., Hashim H., Trukhanov A.V., Korovushkin V.V., Kostishyn V.G. // J. Alloys Compd. 2017. Vol. 698. P. 685.

[13] Elmanov G.N., Chernavskii P.A., Kozlov I.V., Dzhumaev P.S., Kostitsyna E.V., Tarasov V.P., Ignatov A.S., Gudoshnikov S.A. // J. Alloys Compd. 2018. Vol. 741. P. 648-655.

[14] Evstigneeva S., Morchenko A., Trukhanov A., Panina L., Larin V., Volodina N., Yudanov N., Nematov M., Hashim H., Ahmad H. // EPJ Web of Conferences. 2018. Vol. 185. P. 04022.

[15] Zhukov A., Churyukanova M., Kaloshkin S., Semenkova V., Gudoshnikov S., Ipatov M., Talaat A., Blanco J.M., Zhukova V. // J. Alloys Compd. 2015. Vol. 651. P. 718.

[16] Popova A.V., Odintsov V.I., Menshov S.A., Kostitsyna E.V., Tarasov V.P., Zhukova V., Zhukov A., Gudoshnikov S.A. // Intermetallics. 2018. Vol. 99. P. 39.

[17] Kraus L., Frait Z., Pirota K.R., Chiriac H. // J. Magn. Magn. Mater. 2003. Vol. 25. P. 399.

[18] Zhukov A., Talaat A., Churyukanova M., Kaloshkin S., Semenkova V., Ipatov M., Blanco J.M., Zhukova V. // J. Alloys Compd. 2016. Vol. 664. P. 235.

[19] Zhukova V., Ipatov M., Zhukov A., Varga R., Torcunov A., Gonzalez J., Blanco J.M. // J. Magn. Magn. Mater. 2006. Vol. 300. P. 16.

[20] Tian B., Wang L.H., Zhou L.Y. // Adv. Mater. Res. 2009. Vol. 79. P. 1407.

[21] Amirabadizadeh A., Mardani R., Ghanaatshoar M. // J. Alloys Compd. 2015. Vol. 9. P. 372.

[22] Liu J.S., Wang X.D., Chen D.M., Qin F.X., Wang H., Xing D.W., Xue X., Sun J.F. // Phys. Procedia. 2013. Vol. 48. P. 140.

[23] Chiriac H., Ovari T.A. // Prog. Mater. Sci. 1996. Vol. 40. P. 333. 